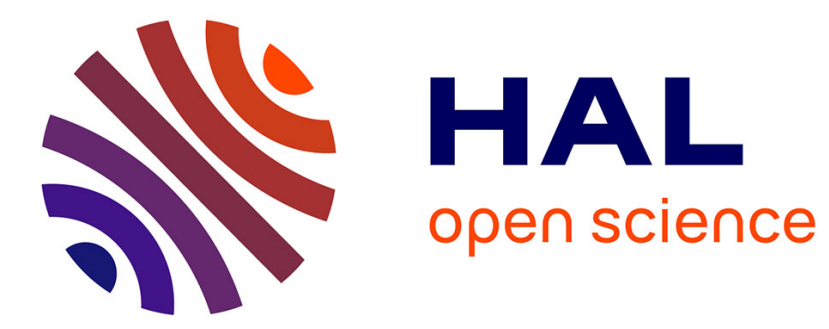

\title{
Time compactness tools for discretized evolution equations and applications to degenerate parabolic PDEs
}

Boris Andreianov

\section{To cite this version:}

Boris Andreianov. Time compactness tools for discretized evolution equations and applications to degenerate parabolic PDEs. Finite Volumes for Complex Applications VI, Jun 2011, Prague, Czech Republic. pp. 21-29, 10.1007/978-3-642-20671-9_3 . hal-00561344v2

\section{HAL Id: hal-00561344 \\ https://hal.science/hal-00561344v2}

Submitted on 24 Feb 2011

HAL is a multi-disciplinary open access archive for the deposit and dissemination of scientific research documents, whether they are published or not. The documents may come from teaching and research institutions in France or abroad, or from public or private research centers.
L'archive ouverte pluridisciplinaire HAL, est destinée au dépôt et à la diffusion de documents scientifiques de niveau recherche, publiés ou non, émanant des établissements d'enseignement et de recherche français ou étrangers, des laboratoires publics ou privés.

\section{다)(1) $(5$}

Distributed under a Creative Commons Attribution - NonCommerciall 4.0 International 


\title{
Time Compactness Tools for Discretized Evolution Equations and Applications to Degenerate Parabolic PDEs
}

Boris Andreianov

\begin{abstract}
We discuss several techniques for proving compactness of sequences of approximate solutions to discretized evolution PDEs. While the well-known AubinSimon kind functional-analytic techniques were recently generalized to the discrete setting by Gallouët and Latché [15], here we discuss direct techniques for estimating the time translates of approximate solutions in the space $L^{1}$. One important result is the Kruzhkov time compactness lemma. Further, we describe a specific technique that relies upon the order-preservation property. Motivation comes from studying convergence of finite volume discretizations for various classes of nonlinear degenerate parabolic equations. These and other applications are briefly described.
\end{abstract}

Key words: time translates, Kruzhkov lemma, order-preservation, finite volumes

\section{Introduction}

Let us think of evolution equations set on a cylindrical domain $Q:=(0, T) \times \Omega \subset$ $\mathbb{R}^{+} \times \mathbb{R}^{N}$. Proving convergence of space-time discretizations of such equations often includes the three following steps : constructing discrete solutions and getting uniform (in appropriate discrete spaces) estimates; extracting a convergent subsequence; writing down a discrete weak formulation (e.g., with discretized test functions) and passing to the limit in the equation in order to infer convergence.

For the first step, obtention of estimates is greatly simplified by preservation, at the discrete level, of the key structure properties of the PDE (such as symmetry, coercivity, monotonicity of the diffusion operators involved; entropy dissipation, for the nonlinear convection operators in the degenerate parabolic case; etc.). For getting discrete a priori estimates test functions are often used, as in the continuous case. Therefore, some analogues of integration-by-parts formulas and chain rules are instrumental for the first step. For the examples we give in this paper, "discrete

Boris Andreianov

CNRS UMR 6623, 16 route de Gray, Besançon, France, e-mail: boris.andreianov@univ-fcomte.fr 
duality" type schemes (mimetic, co-volume, DDFV; see, e.g., [3] and references therein) can be used to guarantee an exact integration-by-parts feature. In contrast, chain rules for derivation in time or in space must be replaced by approximate analogues, often taking the form of convexity inequalities (see, e.g., [4], [3, Sect. 4]).

In this note, we give some insight into convergence proofs for different subclasses of degenerate elliptic-parabolic-hyperbolic PDEs under the general form ${ }^{1}$

$$
u=b(v), w=\varphi(v), \quad u_{t}-\operatorname{div}\left[\mathbf{G}(v)-\mathbf{a}_{\mathbf{0}}(\boldsymbol{\nabla} w)\right]+\psi(v)=f \text { in } Q=(0, T) \times \Omega
$$

with $b(\cdot), \varphi(\cdot), \psi(\cdot)$ continuous $^{2}$ non-decreasing on $\mathbb{R}$, normalized by zero at zero, with a continuous convection flux $\mathbf{G}(\cdot)$ and with $\mathbf{a}_{0}: \mathbb{R}^{N} \rightarrow \mathbb{R}^{N}$ of Leray-Lions type (see e.g. [1, 4]; $p$-laplacian, with $\mathbf{a}_{\mathbf{0}}(\xi)=|\xi|^{p-2} \xi$ is a typical example). For the sake of simplicity, homogeneous Dirichlet boundary condition on $(0, T) \times \partial \Omega$ is taken.

But our main goal is to discuss the second step of the proofs ${ }^{3}$, the one of getting compact ${ }^{4}$ sequences of discrete solutions. For linear problems, the two latter steps are somewhat trivial; indeed, mere functional-analytic bounds would lead to compactness in a weak topology, which is enough to pass to the limit from the discrete to the continuous weak formulation of the PDE. Thinking of nonlinear problems and passage to the limit in nonlinear terms, bounds in functional spaces can be sufficient when combined with basic compact embeddings; but this requires rather strong bounds involving e.g. some estimates of the derivatives. Regarding evolution PDEs of, say, porous medium type, $L^{p}$ bounds are available on the space derivatives but not on the time derivatives (those belong to some negative Sobolev spaces). In this situation, either compactness in an ad hoc strong topology is needed; or the weak compactness coming from uniform boundedness should be combined with some compactification arguments (compensated compactness, Young measures and their reduction, etc.) that exploit in a non-trivial way the particular structure of the PDE in hand (div-curl structure, pseudo-monotonicity, entropy inequalities, etc.).

In this note, we first recall in $\S 2$ the fundamental techniques using only bounds in well-chosen functional spaces (see $[9,11,17,2]$ for the continuous setting; see $[15,12]$ for the corresponding discrete results). In $\S 3$, we present a collection of complementary techniques for estimation of time translates of families of functions that already possess some estimate of space translates. In $\S 4$, we describe one indirect method for proving compactness and convergence of families of approximate solutions. The method heavily exploits the order-preservation property, required both for the PDE in hand and for the approximation scheme in use. Throughout the note, the exposition is motivated and illustrated by applications to approximation of several cases of problem (1) (different cases requiring different approaches).

\footnotetext{
${ }^{1}$ See [5] and references therein for well-posedness theory of such "triply nonlinear" equations. These are mathematical models for porous media, sedimentation, Stefan problem, etc..

${ }^{2}$ Actually, we assume that either these functions are uniformly continuous, or $v$ is bounded a priori.

${ }^{3}$ When the compactification methods strongly utilize a particular structure of the underlying PDE, this step is in fact combined with the third step of passing to the limit.

4 Throughout the note, "compact" actually signifies "relavively compact".
} 


\section{Functional-analytic approach of Aubin-Lions-Dubinskii-Simon}

In the continuous setting, one celebrated result is the Aubin-Lions or Dubinskii lemma ([9] and [11]) and its generalization by Simon [17] (see also Amann [2]). To give an example relevant for the applications we have in mind, let us simply state here that a sequence $\left(u_{h}\right)_{h}$ bounded in $L^{1}\left(0, T ; W^{1,1}(\Omega)\right)$ and such that $\left(\left(u_{h}\right)_{t}\right)_{h}$ is bounded in $L^{1}\left(0, T ; W^{-1,1}(\Omega)\right)$ is relatively compact in $L^{1}(Q)$, cf. [15]. More generally, compactness comes from an a priori bound on $u_{h}$ in some space $L^{p}(0, T, X)$ with $X$ compactly embedded in $L^{1}(\Omega)$ (e.g., $X=W^{1,1}(\Omega)$ ) while the PDE brings information on boundedness of the time derivatives $\left(u_{h}\right)_{t}$ in some space $L^{q}(0, T ; Y)$ where $Y$ can be a subspace of distributions on $\Omega$ equipped with a rather weak topology (e.g., $Y=W^{-1,1}(\Omega)$ ). A discrete version of the Aubin-Simon lemma was recently proposed by Gallouët and Latché in [15]; it is based upon a careful reformulation of estimates in terms of "coherent" families $\left(X_{h}\right)_{h},\left(Y_{h}\right)_{h}$ of discrete spaces.

A related result taken from Simon [17] and Amann [2] uses a bound on fractional time derivatives of $u_{h}$. As it was demonstrated by Emmrich and Thalhammer in [12], this version is quite appropriate in the time-discretized setting. Indeed, time fractional derivatives of order less than $1 / 2$ exist even for piecewise constant functions. Technically, this method involves an indirect estimation of weighted time translates, under a form $\int_{0}^{T} \int_{0}^{T} \frac{\left|u_{h}(t)-u_{h}(s)\right|^{p}}{|t-s|^{1+\sigma p}} d s d t$ with some $p \geq 1$ and $\sigma \in(0,1 / 2)$.

These results only use bounds in functional spaces and very few of the underlying PDE properties. They offer a very wide spectrum of applications; yet they are difficult to apply on degenerate parabolic problems with non-Lipschitz nonlinearities. The difficulty comes from the fact that non-Lipschitz mappings make bad correspondence between linear functional spaces. Yet this difficulty is not a fundamental one; roughly speaking, it is settled by a careful use of translation arguments and of moduli of continuity. This is the object of the next section.

\section{Direct estimation of time translates}

In this section, the compactness question is studied using the one and only space $L^{1}(Q)$. By the Fréchet-Kolmogorov compactness criterion in $L^{1}(Q)$, uniform bounds on space and time translates of $u_{h}$ are needed. In the setting of the present note, the first ones are readily available. The difficulty lies in estimating the time translates as

$$
\forall h \int_{0}^{T-\delta} \int_{\Omega}\left|u_{h}(t+\delta)-u_{h}(t)\right| \leq \omega(\delta) \quad \text { with } \lim _{\delta \rightarrow 0} \omega(\delta)=0,
$$

$\omega(\cdot)$ being a modulus of continuity, uniform in $h$. Here are two ways to obtain (2).

\footnotetext{
${ }^{5}$ Working in an $h$-independent space is an advantage for producing discrete versions of compactness arguments; yet the approach of [15] exhibits a simple and efficient use of $h$-dependent spaces.
} 


\section{A discrete Kruzhkov lemma ${ }^{6}$}

Lemma 1 (Kruzhkov [16]). Assume that the families of functions $\left(u_{h}\right)_{h},\left(F_{h}^{\alpha}\right)_{h, \alpha}$ are bounded in $L^{1}(Q)$ and satisfy $\frac{\partial}{\partial t} u_{h}=\sum_{|\alpha| \leq m} D^{\alpha} F_{h}^{\alpha}$ in $\mathscr{D}^{\prime}(Q)$. Assume that $u_{h}$ can be extended outside $Q$, and one has ${ }^{7}$

$$
\iint_{Q}\left|u_{h}(t, x+\delta)-u_{h}(t, x)\right| d x d t \leq \omega(\delta), \quad \text { with } \lim _{\delta \rightarrow 0} \omega(\delta)=0,
$$

where $\omega(\cdot)$ does not depend on $h$. Then $\left(u_{h}\right)_{h}$ is (relatively) compact in $L^{1}(Q)$.

Clearly, this is an $L_{l o c}^{1}$ compactness result (one can apply the lemma locally in $Q$ ).

For problem (1), the value $m=1$ is relevant, because an $L^{1}$ bound is available for the flux $\left(\mathbf{G}(v)-\mathbf{a}_{\mathbf{0}}(\boldsymbol{\nabla} \varphi(v))\right)$; therefore we limit to this case the discussion of discrete analogues of Lemma 1 . To give an idea of discrete versions of the Kruzhkov lemma ${ }^{8}$, assume we are given a family of meshes of $\Omega$ indexed by their size $h$ and satisfying mild proportionality restrictions (e.g., for the case of two-point flux finite volume schemes as described in [13], one needs for all neighbour volumes $K, L$, $\operatorname{diam}(K)+\operatorname{diam}(L) \leq$ const $d_{K, L}$ uniformly in $\left.h\right)$. Assume that on these meshes, spaces of discrete functions $\mathbb{R}_{h}$ and discrete fields $\left(\mathbb{R}^{N}\right)_{h}$ are defined (each element $u_{h} \in \mathbb{R}_{h}$ or $\mathbf{F}_{h} \in\left(\mathbb{R}^{N}\right)_{h}$ is a piecewise constant on $\Omega$ function reconstructed from the degrees of freedom of the discretization method). Assume we are given discrete gradient and discrete divergence operators $\nabla_{h}$ and $\operatorname{div}_{h}$ mapping between these spaces. Thus all discrete objects (functions, fields, gradient, divergence) are naturally lifted to $L^{1}(Q)$.

Let $\left(\delta_{h}\right)_{h}$ be the associated time steps, let $N_{h}$ be the entire part of $T / \delta_{h}$. Assume that we are given an initial condition $b_{h}^{0}$ and discrete evolution equations under the form

$$
\text { for } n \in\left[1, N_{h}+1\right], \quad \frac{b\left(v_{h}^{n}\right)-b\left(v_{h}^{n-1}\right)}{\delta_{h}}=\operatorname{div}_{h}\left[\mathbf{F}_{h}^{n}\right]+f_{h}^{n} \text { in } \mathbb{R}_{h},
$$

where families $\left(\left(\left(u_{h}^{n}\right)_{n}\right)_{h},\left(\left(f_{h}^{n}\right)_{n}\right)_{h}\right.$ (discrete functions) and $\left(\left(\mathbf{F}_{h}^{n}\right)_{n}\right)_{h}$ (discrete fields) are bounded in $L^{1}(Q)$. Assume that the discrete gradients $\left(\left(\nabla_{h} v_{h}^{n}\right)_{n}\right)_{h}$ are bounded in $L^{1}(Q)$ and that this bound implies a uniform translation bound in space of the family $v_{h}$ (this is true, e.g., when discrete Poincaré inequalities can be proved). Under these assumptions, reproducing at the discrete level the proof [16] of Lemma 1 as it is done in [7, 3], one concludes that the family $\left(b\left(v_{h}\right)\right)_{h}$ is relatively compact in $L^{1}(Q)$. Note that, the case $m \geq 2$ would require more work.

\footnotetext{
${ }^{6}$ There is a strong relation to the method of $\S 2$. The Kruzhkov lemma allows for general moduli of continuity. E.g., for problem (1) with $\varphi=I d$, the Aubin-Lions-Dubinskii-Simon argument can be used if $b(\cdot)$ is Lipschitz continuous (with $X=W_{0}^{1, p}(\Omega)$ ) or Hölder continuous (with a fractional Sobolev space chosen for $X$ ), and the Kruzhkov lemma can be used for any continuous $b(\cdot)$.

${ }^{7}$ In practice, space translation estimates of the kind (3) can be obtained via an estimate of some discrete gradients; notice that estimates of kind (3) are stable upon composing $\left(u_{h}\right)_{h}$ by a function $b(\cdot)$ which is uniformly continuous (as in (1), we mean that $u_{h}=b\left(v_{h}\right)$ ).

${ }^{8}$ Here we give a rather heuristic presentation; see [7] and [3] for two precise formulations covering, e.g., the two-point flux finite volume schemes ([13]) and DDFV schemes ([3] and ref. therein).
} 


\section{A classical technique for the "variational" setting}

Following [1], by "variational" we mean a setting where the solution $w$ is an admissible test function in the weak formulation of the PDE; e.g., (1) can be tested with $w=\varphi(v)$. It typically comes along with a priori estimates that can be reproduced at the discrete level, provided the discretization is somewhat structure-preserving. ${ }^{9}$

The technique of [1] used, in its finite volume version, e.g., in [13, 14, 4], is to integrate ${ }^{10}$ the equation in time from $t$ to $t+\delta$, take $w_{h}(t+\delta)-w_{h}(t)$ for test function, then integrate in $(t, x)$. On problem (1), this leads to a uniform estimate

$$
\forall h>0 \int_{0}^{T-\delta} \int_{\Omega}\left(b\left(v_{h}\right)(t+\delta)-b\left(v_{h}\right)(t)\right)\left(\varphi\left(v_{h}\right)(t+\delta)-\varphi\left(v_{h}\right)(t)\right) \leq \omega(\delta) .
$$

Then Lipschitz continuity of $\varphi \circ b^{-1}$ (resp., of $b \circ \varphi^{-1}$ ) can be used to infer uniform $L^{2}$ time translates of $w_{h}=\varphi\left(v_{h}\right)$ (resp., of $u_{h}=b\left(v_{h}\right)$ ). Yet the $L^{1}$ time translates can be obtained in the case $\varphi \circ b^{-1}$ (resp., $b \circ \varphi^{-1}$ ) is a merely continuous function.

- A technique for $L^{1}$ estimates involving non-Lipschitz nonlinearities (see [4])

Consider the case where $\widetilde{\varphi}:=\varphi \circ b^{-1}$ is a uniformly continuous function (moreover, it is non-decreasing). Let $\pi$ be a concave modulus of continuity for $\varphi \circ b^{-1}, \Pi$ be its inverse, and set $\tilde{\Pi}(r)=r \Pi(r)$. Let $\widetilde{\pi}$ be the inverse of $\widetilde{\Pi}$. Note that $\tilde{\pi}$ is concave, continuous, and $\tilde{\pi}(0)=0$. Set $u^{\delta}=b\left(v_{h}\right)(t+\delta, x)$ and $u=b\left(v_{h}\right)(t, x)$. We have

$$
\int_{Q}\left|\widetilde{\varphi}\left(u^{\delta}\right)-\widetilde{\varphi}(u)\right|=\int_{Q} \widetilde{\pi} \circ \widetilde{\Pi}\left(\left|\widetilde{\varphi}\left(u^{\delta}\right)-\widetilde{\varphi}(u)\right|\right) \leq|Q| \widetilde{\pi}\left(\frac{1}{|Q|} \int_{Q} \widetilde{\Pi}\left(\left|\widetilde{\varphi}\left(u^{\delta}\right)-\widetilde{\varphi}(u)\right|\right)\right) .
$$

Since $\left|\widetilde{\varphi}\left(u^{\delta}\right)-\widetilde{\varphi}(u)\right| \leq \pi\left(\left|u^{\delta}-u\right|\right)$, we have $\Pi\left(\left|\widetilde{\varphi}\left(u^{\delta}\right)-\widetilde{\varphi}(u)\right|\right) \leq\left|u^{\delta}-u\right|$ and

$\widetilde{\Pi}\left(\left|\widetilde{\varphi}\left(u^{\delta}\right)-\widetilde{\varphi}(u)\right|\right)=\Pi\left(\left|\widetilde{\varphi}\left(u^{\delta}\right)-\widetilde{\varphi}(u)\right|\right)\left|\widetilde{\varphi}\left(u^{\delta}\right)-\widetilde{\varphi}(u)\right| \leq\left|u^{\delta}-u\right|\left|\widetilde{\varphi}\left(u^{\delta}\right)-\widetilde{\varphi}(u)\right|$.

Therefore, (5) implies an $L^{1}$ estimate of the kind (2) on $w_{h}=\varphi\left(v_{h}\right)$ :

$$
\int_{Q}\left|w_{h}(t+\delta)-w_{h}(t)\right| \leq|Q| \widetilde{\pi}\left(\frac{1}{|Q|} \int_{Q}\left|u^{\delta}-u\right|\left|\widetilde{\varphi}\left(u^{\delta}\right)-\widetilde{\varphi}(u)\right|\right)=|Q| \widetilde{\pi}\left(\frac{1}{|Q|} \omega(\delta)\right) .
$$

- Use of contraction arguments and absorption terms (see [8])

Let us mention one more possibility for getting estimates of kind (2) for (1), which takes advantage of the monotonicity of $\psi(\cdot)$. Assume $\varphi=I d$ in (1); to shorten the arguments, assume $f=0$. Then $L^{1}$ translates in time of $u_{h}=b\left(v_{h}\right)$ can be estimated with every of the two preceding methods, the Kruzhkov lemma and a direct estimation of translates with variational techniques. This makes $\left(b\left(v_{h}\right)\right)_{h}$ relatively compact; yet, when $b^{-1}(\cdot)$ is discontinuous, no information on compactness of $\left(v_{h}\right)_{h}$ is

\footnotetext{
${ }^{9}$ Notice that for evolution PDEs governed by accretive in $L^{1}(\Omega)$ operators, of which (1) is an example, time-implicit discretizations are better suited for structure preservation. Use of numerical schemes in space that possess a kind of discrete duality (mimetic, co-volume, DDFV schemes, etc.) enables getting discrete estimates analogous to the continuous ones. For notions of solution involving some version of chain rule (e.g., entropy, renormalized solutions) orthogonality assumption on the meshes and isotropy assumption on the diffusion operator may be needed, see e.g. [4].

${ }^{10}$ Here, for the sake of simplicity, we stick to the terminology and notation of the continuous case.
} 
obtained this way. Now, let us use the translation (in time) invariance of the equation and the $L^{1}$ contraction property ${ }^{11}$ natural for (1). This yields the estimate (see [8])

$$
\int_{\Omega}\left|b\left(v_{h}^{\delta}\right)-b\left(v_{h}\right)\right|(T-\delta)+\int_{s}^{T-\delta} \int_{\Omega}\left|\psi\left(v_{h}^{\delta}\right)-\psi\left(v_{h}\right)\right| \leq \int_{\Omega}\left|b\left(v_{h}^{\delta}\right)(s)-b\left(v_{h}\right)(s)\right|
$$

for all $s \in(0, T-\delta)$, where $v_{h}^{\delta}(t)=v_{h}(t+\delta)$. Integrating in $s>\alpha>0$, using the time translation bound for $\left(b\left(v_{h}\right)\right)_{h}$ we get an $L^{1}((\alpha, T) \times \Omega)$ estimate of time translates of $\psi\left(v_{h}\right)$. If $\psi(\cdot)$ is strictly increasing, this is enough for $L_{l o c}^{1}$ compactness of $\left(v_{h}\right)_{h}$.

\section{Applications to (1) and some other parabolic PDEs}

- Application to a parabolic-hyperbolic PDE (see [4])

For problem (1) with $b=I d$, provided $L^{p}(Q)$ estimates of the discrete gradient of $\varphi\left(v_{h}\right)$ are available, space translates of $\varphi\left(v_{h}\right)$ (and the functions $\varphi\left(v_{h}\right)$ themselves) can be estimated uniformly, and an estimate of the form (5) can be obtained. Then the above technique for exploiting (5) assesses the $L^{1}(Q)$ compactness of $\left(\varphi\left(v_{h}\right)\right)_{h}$, which is a first step of the convergence proof for this problem (see [4] $)^{12}$.

- Application to an elliptic-parabolic PDE with the structure condition (see [3]) Assume $\varphi=I d$. Estimate (5) controls the $L^{1}$ time translates of $b\left(v_{h}\right)$ similarly to what was described above ${ }^{13}$. If the structure condition $\mathbf{G}(v)=\mathbf{F}(b(v))$ is satisfied, compactness of $\left(b\left(v_{h}\right)\right)_{h}$ is enough to pass to the limit, see [1] (cf. [10] and $\S 4$ ).

- Application to a cross-diffusion system (see [7])

The following kind of models comes from population dynamics:

$$
\left\{\begin{array}{l}
u_{t}-D_{1} \Delta u-\operatorname{div}((u+v) \nabla u+u \boldsymbol{\nabla} v)=u\left(a_{1}-b_{1} u-c_{1} v\right), \\
v_{t}-D_{2} \Delta v-\operatorname{div}(v \boldsymbol{\nabla} u+(u+v) \boldsymbol{\nabla} v)=v\left(a_{2}-b_{2} u-c_{2} v\right) .
\end{array}\right.
$$

Natural estimates for approximate solutions of (7) are $L^{2}$ bounds on $\sqrt{1+u+v} \nabla u$, $\sqrt{1+u+v} \nabla v$; this gives only an $L^{4 / 3}$ bound on the diffusion fluxes in (7), thus we are not in a variational setting ${ }^{14}$. Therefore for a proof of convergence of finite volume approximations of the kind [13], the Kruzhkov lemma was used in [7] ${ }^{15}$.

- Application to convergence of some linearized implicit schemes (see [6])

In [6], discretization of the simplified version of cardioelectrical bidomain model:

$$
\left\{\begin{array}{l}
v_{t}-\operatorname{div}\left[\mathbf{M}_{i}(\cdot) \boldsymbol{\nabla} u_{i}\right]+H(v)=I_{a p}(\cdot), \\
v_{t}+\operatorname{div}\left[\mathbf{M}_{e}(\cdot) \boldsymbol{\nabla} u_{e}\right]+H(v)=I_{a p}(\cdot),
\end{array} \quad v=u_{i}-u_{e},\right.
$$

was considered; here, the "ionic current" $H(\cdot)$ is a cubic polynomial. This nonlinear

\footnotetext{
${ }^{11}$ Discrete version of (6) (see [8]) assumes the $L^{1}$ contraction property (linked to order preservation via the Crandall-Tartar lemma) is preserved at the discrete level. Estimate (6) is exploited in $\S 4$.

${ }^{12}$ For Lipschitz $\varphi(\cdot)$, also the Aubin-Lions-Dubinskii-Simon and Kruzhkov lemmas could be used. For general $\varphi(\cdot)$, the author thinks that neither of these lemmas can replace the direct use of (5).

${ }^{13}$ Alternatively, the Kruzhkov lemma can be used in a straightforward way, see [3].

${ }^{14}$ From the practical point of view, e.g. the first equation cannot be tested with $u(t+\delta)$.

${ }^{15}$ Alternatively, the discrete Aubin-Lions-Dubinskii-Simon lemma (see [15]) could be used here.
} 
reaction term brings an estimate of $v H(v)$ which bounds $v$ in $L^{4}(Q)$. Time-implicit DDFV discretization of (8) preserves this structure; then the problem falls into the "variational" framework ${ }^{16}$ and time translates can be estimated like in $[1,13,14]$. From the practical point of view, it is important to accelerate computations, and to consider a linearized method where the discretization of the reaction term $H(v)$ is not fully implicit. Unfortunately, for theoretical analysis $L^{4}$ estimate for $v_{h}$ is not available any more; only a weaker estimate can be obtained with interpolation arguments. In [6], we applied the Kruzhkov lemma to exploit this weaker estimate ${ }^{17}$.

\section{Advanced use of the underlying PDE features}

Often mere functional-analytic bounds are not enough, but additional constraints coming from the particular structure of the approximated PDE may permit an indirect compactness/convergence proof. E.g., for the parabolic-hyperbolic PDE (1) (case $b=I d$ ) we proved the compactness of $\left(\varphi\left(v_{h}\right)\right)_{h}$ in $\S 3$. The two final steps (see [4]; see also [14]) exploit fine PDE tools. First, the Minty argument (see, e.g., [1]) is used for $\left(\mathbf{a}_{\mathbf{0}}\left(\boldsymbol{\nabla}_{h} \varphi\left(v_{h}\right)\right)_{h}\right.$; second, the "nonlinear weak-* convergence" $([13,14,4])$ for $\left(v_{h}\right)_{h}$ is upgraded to strong convergence using entropy inequalities for (1).

Let us show how one very delicate case of (1), see [10], can be treated indirectly.

\section{Compactness from monotone penalization and order-preservation}

For getting (6), we already used the order-preservation structure for (1). Its further use, in conjunction with penalization, may lead to the following convergence proof.

\section{- The structure needed for compactification}

Assume that one can prove uniqueness of a solution to a PDE $\left(E q^{0}\right)$ under study. Assume that $\left(E q^{0}\right)$ can be embedded "continuously" into a family $\left(E q^{\varepsilon}\right)$ of perturbed PDEs having the property that $v_{h}^{\varepsilon_{1}} \leq v_{h}^{\varepsilon_{2}}$ when $\varepsilon_{1} \leq \varepsilon_{2}$, where $v_{h}^{\varepsilon_{1}}, v_{h}^{\varepsilon_{2}}$ are the associated discrete solutions. Continuity in $\varepsilon \in[-1,1]$ means, we assume that limits as $\varepsilon \rightarrow 0$ (if they exist) of exact solutions $v^{\varepsilon}$ of $\left(E q^{\varepsilon}\right)$ solve the limit equation $\left(E q^{0}\right)$.

Assume that for $\varepsilon \neq 0$, the corresponding sequence $\left(v_{h}^{\varepsilon}\right)_{h}$ is well defined and it converges to an exact solution $v^{\varepsilon}$ of $\left(E q^{\varepsilon}\right)$. Then solutions $\left(v_{h}^{0}\right)_{h}$ to the discretized equation $\left(E q^{0}\right)$ converge a.e., as $h \rightarrow 0$, to the unique solution of $\left(E q^{0}\right)$. Indeed, write

$$
v_{h}^{-1} \leq v_{h}^{-1 / 2} \leq \ldots \leq v_{h}^{-1 / m} \leq \ldots \leq v_{h}^{0} \leq \ldots \leq v_{h}^{1 / m} \leq \ldots \leq v_{h}^{1 / 2} \leq v_{h}^{1},
$$

and pass to the limit as $h \rightarrow 0$ to define $v^{ \pm 1 / m}:=\lim _{h \rightarrow 0} v_{h}^{ \pm 1 / m}$ (up to extraction of a subsequence) solution to $\left(E q^{ \pm 1 / m}\right)$; then, (9) is inherited at the limit (except that $\left(v_{h}^{0}\right)_{h}$ may not have a limit). By monotonicity, we can define $\underline{v}:=\lim _{m \rightarrow \infty} v^{-1 / m}$ and $\bar{v}:=\lim _{m \rightarrow \infty} v^{1 / m}$; furthermore, we have $\underline{v} \leq \liminf _{h \rightarrow 0} v_{h}^{0} \leq \limsup _{h \rightarrow 0} v_{h}^{0} \leq \bar{v}$. Both $\underline{v}, \bar{v}$ solve $\left(E q^{0}\right)$. Thus, by uniqueness, $\left(v_{h}^{0}\right)_{h}$ converges to $\underline{v} \equiv \bar{v}$ the solution of $\left(E q^{0}\right)$.

${ }^{16}$ Indeed, we have $v_{h}$ bounded in $L^{4}(Q)$ and $H\left(v_{h}\right)$ is bounded in $L^{4 / 3}(Q)=\left(L^{4}(Q)\right)^{*}$.

${ }^{17}$ A discrete Aubin-Lions-Dubinskii-Simon argument could have been applied as well. 
- Application to an elliptic-parabolic PDE without the structure condition (see [8]) We assume that $\varphi=I d, \psi=0$ in (1). We have seen that compactness of $\left(b\left(v_{h}\right)\right)_{h}$ can be established, e.g., with the Kruzhkov lemma. Under the structure condition $\mathbf{G}(v)=\mathbf{F}(b(v))$, this is enough to pass to the limit in the equation. But in general (see [10]) one lacks control of time oscillations of $\mathbf{G}\left(v_{h}\right)$, and the method of [1] fails. Yet it is enough to add penalization term of the form $\psi^{\varepsilon}(v)=\varepsilon\left(\arctan v \mp \frac{\pi}{2} \operatorname{sign} \varepsilon\right)$ to get into the setting where (6) can be exploited to control discrete solutions $\left(v_{h}^{\varepsilon}\right)_{h}$ and to pass to the limit, as $h \rightarrow 0$, for the $\psi^{\varepsilon}$-penalized equation $\left(1^{\varepsilon}\right)$. The orderpreservation assumptions of the above method being fulfilled due to the choice of $\psi^{\varepsilon}$, we get convergence of $\left(v_{h}\right)_{h}$ in the cases where uniqueness for (1) can be shown.

Acknowledgements The author thanks E. Emmrich for discussions on the above techniques.

\section{References}

1. H.W. Alt and S. Luckhaus. Quasilinear elliptic-parabolic differential equations. Mat.Z., (1983), 183:311-341.

2. H. Amann. Compact embeddings of vector-valued Sobolev and Besov spaces. Glasnik Matematički, (2000), 35:161-177.

3. B. Andreianov, M. Bendahmane and F. Hubert. On 3D DDFV discretization of gradient and divergence operators. II. Discrete functional analysis tools and applications to degenerate parabolic problems. Preprint HAL, (2011), http://hal.archives-ouvertes.fr/hal-00567342

4. B. Andreianov, M. Bendahmane, and K.H. Karlsen. Discrete duality finite volume schemes for doubly nonlinear degenerate hyperbolic-parabolic equations. J. Hyp. Diff. Eq., (2010), 7:1-67.

5. B. Andreianov, M. Bendahmane, K.H. Karlsen and S. Ouaro. Well-posedness results for triply nonlinear degenerate parabolic equations. J. Diff. Eq., (2009), 247(1):277-302.

6. B. Andreianov, M. Bendahmane, K.H. Karlsen and Ch. Pierre. Convergence of Discrete Duality Finite Volume schemes for the macroscopic bidomain model of the heart electric activity. Netw. Het. Media, (2011), to appear; available at http://hal.archives-ouvertes.fr/hal-00526047

7. B. Andreianov, M. Bendahmane and R. Ruiz Baier. Analysis of a finite volume method to solve a cross-diffusion population system. Math. Models Meth. Appl. Sci., (2011), to appear.

8. B. Andreianov and P. Wittbold. Convergence of approximate solutions to an elliptic-parabolic equation without the structure condition. Preprint, (2011).

9. J.-P. Aubin. Un théorème de compacité. (French) C.R. Acad. Sc. Paris, (1963), 256:5042-5044

10. Ph. Bénilan and P. Wittbold. Sur un problème parabolique-elliptique. (French) M2AN Math. Modelling and Num. Anal., (1999), 33(1):121-127.

11. J.A. Dubinskii. Weak convergence for elliptic and parabolic equations. (Russian) Math. USSR Sbornik, (1965), 67:609-642.

12. E. Emmrich and M. Thalhammer. Doubly nonlinear evolution equations of second order: Existence and fully discrete approximation. J. Diff. Eq., (2011), to appear.

13. R. Eymard, T. Gallouët, and R. Herbin. Finite Volume Methods. Handbook of Numerical Analysis, Vol. VII (2000). P. Ciarlet, J.-L. Lions, eds., North-Holland.

14. R. Eymard, T. Gallouët, R. Herbin and A. Michel. Convergence of a finite volume scheme for nonlinear degenerate parabolic equations. Numer. Math., (2002), 92(1):41-82.

15. T. Gallouët and J.-C. Latché. Compactness of discrete approximate solutions to parabolic PDEs - Application to a turbulence model. Comm. on Pure and Appl. Anal., (2011), to appear.

16. S.N. Kruzhkov. Results on the nature of the continuity of solutions of parabolic equations and some of their applications. Mat. Zametki (Math. Notes), (1969), 6(1):517-523.

17. J. Simon. Compact sets in the space $L^{p}(0, T ; B)$. Ann. Mat. Pura ed Appl., (1987), 146:65-96. 